\title{
A NONCOMPACT CHOQUET THEOREM
}

\author{
G. A. EDGAR
}

ABSTRACT. The following noncompact analog of Choquet's theorem is proved. Let $E$ be a Banach space with the Radon-Nikodým property, let $C$ be a separable, closed, bounded, convex subset of $E$, and let $a$ be a point in $C$. Then there is a probability measure $\mu$ on the universally measurable sets in $C$ such that $a$ is the barycenter of $\mu$ and the set of extreme points of $C$ has $\mu$-measure 1 .

The Krein-Milman theorem states that every compact convex set $C$ in a locally convex space is the closed convex hull of its extreme points. Choquet's theorem (see [9]) is a strengthening of the Krein-Milman theorem in the metrizable case: if such a set $C$ is metrizable, then every point in $C$ is the barycenter of a probability measure concentrated on the extreme points of $C$. Roughly speaking, this says that every point of $C$ is an average of extreme points.

C. Bessaga and A. Pelczyński [1] proved the following Krein-Milmanlike theorem: every closed bounded convex set $C$ in a separable dual Banach space is the closed convex hull of its extreme points. R. Bourgin [2] attempted to obtain a corresponding Choquet-type theorem.

J. Lindenstrauss [10, Theorem 2] has recently improved the BessagaPełczyński theorem: every closed bounded convex set $C$ in a Banach space with the Radon-Nikodým property is the closed convex hull of its extreme points. (According to [3], if a Banach space is a separable dual, or is weakly complete and has a separable dual, or is reflexive, then it has the RadonNikodým property; thus these three kinds of spaces are covered by the Lindenstrauss theorem.) In the present paper, we prove the corresponding Choquet-type theorem: if such a set $C$ is separable, then every point of $C$ is the barycenter of a probability measure concentrated on the extreme points of $C$.

Presented to the Society, January 23, 1975; received by the editors March 29, 1974.

AMS (MOS) subject classifications (1970). Primary 46B99; Secondary 46G10, 28A40, 60G45.

Key words and phrases. Choquet's theorem, Radon-Nikodým property, extreme measure, vector-valued martingale. 
The idea of the proof is very simple. We begin with a point $a$ in $C$. If $a$ is an extreme point, we are done; if not, then it can be written as a midpoint $a=1 / 2\left(a_{1}+a_{2}\right)$ of two distinct points of $C$. Continue in this manner, at each stage writing all nonextreme points in the average as midpoints of two distinct points. "Eventually" all points in the average will be extreme points. The complications in the proof arise when we try to make precise the word "eventually" in the previous sentence. The process must be repeated into the transfinite before it necessarily reaches the extreme points. The proof is couched in probabilistic language; the relevant terminology and notation can be found in [3].

1. Preliminaries. If $C$ is a convex set, and $x \in C$, then $x$ is called an extreme point of $C$ iff there do not exist distinct points $y, z \in C$ with $x=1 / 2(y+z)$. The set of all extreme points of $C$ will be denoted by ex $C$.

Recall [3] that if a Banach space has the Radon-Nikodým property, then bounded Bochner-integrable martingale sequences converge a.s. It follows from this that a bounded Bochner-integrable martingale on any countable ordinal converges a.s.

If $\mathcal{F}$ is a sigma-algebra on a set $G$, then a subset $A$ of $G$ is called universally measurable [7, II.28.c] with respect to $\mathcal{F}$ iff, for every probability measure $\mu$ on $\mathcal{F}$, there exist $A_{1}, A_{2} \in \mathcal{F}$ with $A_{1} \subseteq A \subseteq A_{2}$ and $\mu\left(A_{1}\right)=$ $\mu\left(A_{2}\right)$. The set of all universally measurable sets is called the universal completion of $\mathfrak{F}$, and is denoted by $\mathcal{U}(\mathcal{F})$. Note that $\mathcal{U}(\mathcal{U}(\mathcal{F}))=\mathcal{U}(\mathcal{F})$. $A$ sigma-algebra $\mathfrak{F}$ is called universal iff $\mathcal{U}(\mathfrak{F})=\mathscr{F}$. A subset of a metric space $C$ is simply called universally measurable if it belongs to $\mathcal{U}(\mathcal{B})$, where $B$ is the set of Borel sets of $C$. If $R$ is a set of subsets of a set $G$, we will write $\mathcal{U}(\mathscr{R})$ for the universal completion of the sigma-algebra generated by $R$. Finally, if $f: G_{1} \rightarrow G_{2}$ is measurable with respect to the sigmaalgebras $\mathcal{F}_{1}$ and $\mathcal{F}_{2}$, then it is measurable with respect to $\mathcal{U}\left(\mathcal{F}_{1}\right)$ and $\mathcal{U}\left(\mathcal{F}_{2}\right)$.

\section{Main theorem.}

Theorem. Let $E$ be a Banach space with the Radon-Nikodym property, and let $C$ be a closed, bounded, separable, convex set in $E$. Then for every $a \in C$, there is a probability measure $\mu$ on the universally measurable sub. sets of $C$ such that $a=\int x d \mu(x)$ as a Bochner integral, and $\mu(\operatorname{ex} C)=1$. In particular, $C$ is the closed convex hull of ex $C$.

Proof. Let $\Omega$ denote the first uncountable ordinal and $G=\{0,1\}^{\Omega}$ the 
set of $\Omega$-sequences of 0 's and 1's. For each ordinal $a<\Omega$, let $R_{\alpha}=\{p \epsilon$ $G: p(\alpha)=0\}$. Put the product measure $P$ on $G$ from the measure which assigns $1 / 2$ to each element of $\{0,1\}$. The sigma-algebra on $G$ will be $\mathcal{F}=$ $\left.\mathcal{U} R_{\beta}: \beta<\Omega\right\}$, the universal completion of the product sigma-algebra. For $\alpha<\Omega$, define the sub-sigma-algebra $\mathfrak{F}_{\alpha}=\mathcal{U}\left\{R_{\beta}: \beta<\alpha\right\}$. If $\alpha \leq \beta<\Omega$, then $\mathcal{F}_{a} \subseteq \mathcal{F}_{\beta}$. In particular $\mathfrak{F}_{0}=\{\varnothing, G\}$.

Now $C$ is a Polish space, so $D=\{\langle x, y\rangle \in C \times C: x \neq y\}$ is also a Polish space. Define $h: D \rightarrow C$ by $h(x, y)=1 / 2(x+y)$. Thus $h$ is continuous, so (by an easy corollary [6, Corollary 3$]$ of von Neumann's selection theorem [8, Lemma 5]) its image $C \backslash$ ex $C$ is a universally measurable (indeed, analytic) set, and there is a universally measurable section $f: C \backslash$ ex $C \rightarrow D$ of $h$. Define $f$ on ex $C$ by $f(x)=\langle x, x\rangle$, so that $f$ is a universally measurable function $C \rightarrow C \times C$. Let $f_{0}, f_{1}$ be the two components of $f$, so that $f(x)=\left\langle f_{0}(x), f_{1}(x)\right\rangle$. Thus $f_{0}$ and $f_{1}$ are universally measurable functions $C \rightarrow C$ and for all $x \in C$, we have $x=1 / 2\left(f_{0}(x)+f_{1}(x)\right)$. Also, $f_{0}(x)=x$ if and only if $f_{1}(x)=x$ if and only if $x \in$ ex $C$. (L. H. Loomis has used a device similar to this in connection with the compact Choquet theorem [5].)

We will next define inductively a random variable $X_{a}: G \rightarrow C$ for each ordinal $\alpha<\Omega$, so that

$$
E\left[X_{\gamma} \mid F_{\beta}\right]=X_{\beta} \quad \text { for } \beta \leq \gamma<\Omega .
$$

Define $X_{0}(p)=a$ for all $p \in G$. Then (1) is true for $\gamma=0$. If $X_{a}$ is defined and (1) is true for $\gamma=\alpha$, define

$$
X_{a+1}(p)=f_{p(\alpha)}\left(X_{\alpha}(p)\right) .
$$

Now $\{p \in G: p(\alpha)=0\}=R_{a} \in \mathcal{F}_{a+1}$ and $X_{a}$.is $\mathcal{F}_{\alpha}$-measurable, so $X_{a+1}$ is

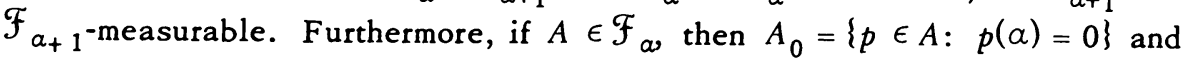
$A_{1}=\{p \in A: p(\alpha)=1\}$ each have half the measure of $A$, so

$$
\begin{aligned}
\int_{A} X_{a+1} d P & =\int_{A_{0}} X_{a+1} d P+\int_{A_{1}} X_{a+1} d P \\
& =\int_{A_{0}} f_{0} \circ X_{a} d P+\int_{A_{1}} f_{1} \circ X_{a} d P \\
& =\frac{1}{2} \int_{A} f_{0} \circ X_{\alpha} d P+\frac{1}{2} \int_{A} f_{1} \circ X_{a} d P=\int_{A} X_{a} d P,
\end{aligned}
$$

i.e., $E\left[X_{a+1} \mid \mathcal{F}_{a}\right]=X_{a}$. Finally, if $\beta<\alpha$, then

$$
E\left[X_{a+1} \mid \mathscr{F}_{\beta}\right]=E\left[E\left[X_{a+1} \mid \mathscr{F}_{a}\right] \mid \mathscr{F}_{\beta}\right]=E\left[X_{a} \mid F_{\beta}\right]=X_{\beta} .
$$


Thus (1) holds for $\gamma=\alpha+1$. Now suppose that $\alpha$ is a limit ordinal, that $X_{\gamma}$ is defined for $\gamma<\alpha$, and that (1) holds for $\gamma<\alpha$. By (1), $\left\langle X_{\gamma}\right\rangle_{\gamma<\alpha}$ is a martingale, so $X_{a}=\lim _{\gamma<\alpha} X_{\gamma}$ exists a.s. Now $X_{a}$ is measurable with respect to $\mathcal{F}_{\alpha}$, so (1) holds with $\beta=\gamma=\alpha$. If $\beta<\alpha$, then for $A \in \mathcal{F}_{\beta}$, we have, by the dominated convergence theorem for Bochner integrals [4, Theorem 7.5.9],

$$
\int_{A} X_{\alpha} d P=\lim _{\gamma<\alpha} \int_{A} X_{\gamma} d P=\lim _{\gamma<\alpha} \int_{A} X_{\beta} d P=\int_{A} X_{\beta} d P,
$$

so that (1) holds for $\beta<\alpha=\gamma$. This completes the definition of the $X_{a}$ by induction. Note that $\beta=0$ in (1) yields

$$
\int X_{a} d P=a
$$

for all $\alpha<\Omega$.

We will next prove that there is an ordinal $\eta<\Omega$ such that $X_{\beta}=X_{\eta}$ a.s. for all $\beta \geq \eta$. Let $\phi: C \rightarrow \mathbf{R}$ be a bounded, universally measurable, strictly convex function. (In fact, since $C$ is separable, there is such a $\phi$ which is continuous; cf. [9, p. 20].) Define $\psi: C \rightarrow \mathbf{R}$ by $\psi(x)=1 / 2 \phi\left(f_{0}(x)\right)+$ $1 / 2 \phi\left(f_{1}(x)\right)$. Then $\psi(x) \geq \phi(x)$ with equality if and only if $x \in$ ex $C$. Now

$$
\begin{aligned}
\int \phi \circ X_{\alpha} d P & \leq \int \psi \circ X_{a} d P \\
& =\frac{1}{2} \int \phi \circ f_{0} \circ X_{a} d P+\frac{1}{2} \int \phi \circ f_{1} \circ X_{a} d P=\int \phi \circ X_{a+1} d P .
\end{aligned}
$$

Thus $\left\langle\int \phi \circ X_{a} d p\right\rangle_{\alpha<\Omega}$ is a nondecreasing $\Omega$-sequence of real numbers, so it is eventually constant, i.e., there is an ordinal $\eta<\Omega$ with $\int \phi \circ X_{\beta} d P=$ $\int \phi \circ X_{\eta} d P$ for all $\beta \geq \eta$. Thus $\int \psi \circ X_{\eta} d P=\int \phi \circ X_{\eta} d P$, so $\psi \circ X_{\eta}=\phi \circ X_{\eta}$ a.s., and thus $X_{\eta}(p) \in \operatorname{ex} C$ a.s. But if $X_{\eta}(p) \in \operatorname{ex} C$, then $X_{\beta}(p)=X_{\eta}(p)$ for all $\beta \geq \eta$, so $X_{\beta}=X_{\eta}$ a.s. for all $\beta \geq \eta$.

Let $\mu$ be the distribution of $X_{\eta}$, i.e., for every universally measurable subset $T$ of $C$, define $\mu(T)=P\left\{p \in G: X_{\eta}(p) \in T\right\}$. Thus $\mu$ is a probability measure, $\mu(\operatorname{ex} C)=1$, and $\int x d \mu(x)=\int X_{\eta} d P=a$ by (2).

3. Additional remarks. Clearly the methods used to prove the theorem can be used in other circumstances as well. The three crucial ingredients are: (i) the section $f$, (ii) the martingale convergence theorem, and (iii) the strictly convex function $\phi$. All of them are available, for example, in the situation of Choquet's theorem itself (i.e., a compact, convex, metrizable subset of a locally convex space). Now, (i) is available for any Polish convex set in a locally convex space. Also, (iii) is available in any strictly convex- 
ifiable Banach space (let $\phi$ be the square of a strictly convex norm). The reader can probably think of other situations where one or more of the three conditions is satisfied.

I have been unable to generalize the theorem to nonseparable sets $C$. The difficulty is in obtaining a replacement for condition (i). It is conceivable that for nonseparable $C$, the set ex $C$ might fail to be universally measurable; in that case, some generalized notion of a measure being supported by ex $C$ may be needed, as in the compact case $[9, \$ 4]$. Or, some smaller sigma-algebra may be appropriate, such as the sigma-algebra generated by the dual space $E^{*}$ of the Banach space $E$; measures on this sigma-algebra need not have separable support.

\section{REFERENCES}

1. C. Bessaga and A. Pełczyński, On extreme points in separable conjugate spaces, Israel J. Math. 4 (1966), 262-264. MR 35 \#2126.

2. R. D. Bourgin, Barycenters of measures on certain noncompact convex sets, Trans. Amer. Math. Soc. 154 (1971), 323-340. MR $42 \# 6582$.

3. S. D. Chatterji, Martingale convergence and the Radon-Nikody'm theorem in Banach spaces, Math. Scand. 22 (1968), 21-41. MR 39 \#7645.

4. E. Hille, Methods in classical and functional analysis, Addison-Wesley, Reading, Mass., 1972.

5. L. H. Loomis, Dilations and extremal measures, Advances in Math. (to appear).

6. A. Lubin, Extensions of measures and the von Neumann selection theorem, Proc. Amer. Math. Soc. 43 (1974), 118-122.

7. P.-A. Meyer, Probability and potentials, Blaisdell, Waltham, Mass., 1966. MR 34 \#5119.

8. J. von Neumann, On rings of operators. Reduction theory, Ann. of Math. (2) 50 (1949), 401-485. MR 10, 548.

9. R. R. Phelps, Lectures on Choquet's theorem, Van Nostrand, Princeton, N. J., 1966. MR $33 \# 1690$.

10. - Dentability and extreme points in Banach spaces, J. Functional Analysis 17 (1974), 78-90.

DEPARTMENT OF MATHEMATICS, NORTHWESTERN UNIVERSITY, EVANSTON, ILLINOIS 60201 